\title{
Oxidized carbonized cellulose coated filters for environmental contaminant adsorption and detection
}

Karin H. Adolfsson, ${ }^{a}$ Giuseppe Melilli, ${ }^{a}$ and Minna Hakkarainen ${ }^{\mathrm{a}, *}$

a Department of Fibre and Polymer Technology, KTH Royal Institute of Technology,

Teknikringen 58, SE-100 44 Stockholm, Sweden.

*Corresponding author: Minna Hakkarainen. E-mail address: minna@kth.se

10 pages, 8 figures, 5 tables 


\section{Table of Contents}

1. Electron microscopy images of oxidized carbonized cellulose (OCC) 3

2. XPS analysis of OCC and filters 4

3. Cationization reaction $\quad 6$

4. OCC coated filters $\quad 9$

5. Additional SALDI-MS measurements 11 
1. Electron microscopy images of oxidized carbonized cellulose
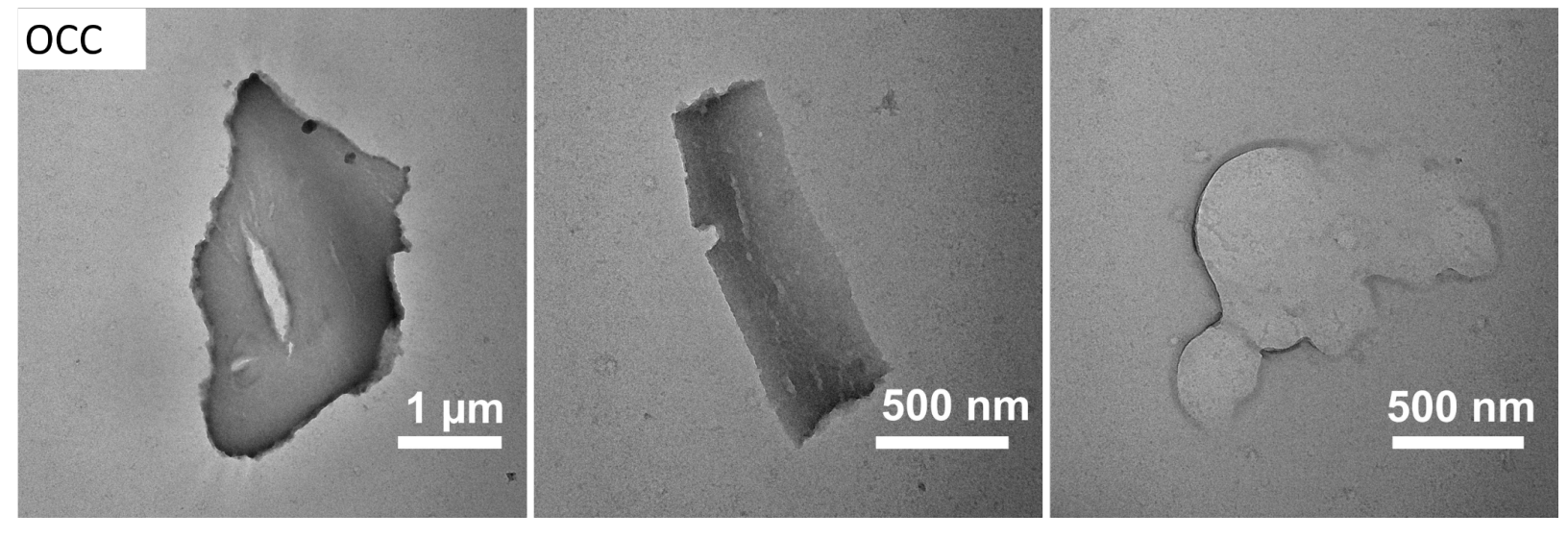

Figure S1. TEM images of OCC. 


\section{XPS analysis of OCC and filters}

Table S1. Details of XPS narrow scan for OCC and filters with suggested assignments.

\begin{tabular}{|c|c|c|c|c|c|c|c|c|c|c|c|c|}
\hline C 1s & & $\mathrm{OCC}$ & & & C & & & C150 & & & $=1500$ & \\
\hline Sample & $\begin{array}{l}\mathrm{BE}, \\
\mathrm{eV}\end{array}$ & $\begin{array}{c}\text { FWHM, } \\
\mathrm{eV}\end{array}$ & $\begin{array}{l}\mathrm{AC}, \\
\text { at.\% }\end{array}$ & $\begin{array}{l}\mathrm{BE}, \\
\mathrm{eV}\end{array}$ & $\begin{array}{c}\text { FWHM, } \\
\text { eV }\end{array}$ & $\begin{array}{l}\text { AC, } \\
\text { at.\% }\end{array}$ & $\begin{array}{l}B E, \\
e V\end{array}$ & $\begin{array}{c}\text { FWHM, } \\
\text { eV }\end{array}$ & $\begin{array}{l}\text { AC, } \\
\text { at.\% }\end{array}$ & $\begin{array}{l}\mathrm{BE}, \\
\mathrm{eV}\end{array}$ & $\begin{array}{c}\text { FWHM, } \\
\text { eV }\end{array}$ & $\begin{array}{l}\text { AC, } \\
\text { at.\% }\end{array}$ \\
\hline $\mathrm{C}-\mathrm{C}, \mathrm{H}$ & 285.0 & 1.1 & 45.77 & 285.0 & 1.05 & 24.27 & 285.0 & 0.95 & 41.68 & 285.0 & 1.15 & 23.55 \\
\hline $\mathrm{C}-\mathrm{O}-\mathrm{C}$ & 285.9 & 1.25 & 13.93 & & & & & & & & & \\
\hline $\mathrm{C}-\mathrm{OH}, \mathrm{N}$ & 287.2 & 1.15 & 6.88 & 286.8 & 1.1 & 31.95 & 286.7 & 1.15 & 24.24 & 286.8 & 1.1 & 32.52 \\
\hline $\mathrm{O}-\mathrm{C}-\mathrm{O}$ & 288.2 & 1.1 & 3.5 & 288.3 & 1.1 & 7.71 & 288.2 & 0.95 & 6.01 & 388.2 & 1.2 & 8.82 \\
\hline $\mathrm{O}=\mathrm{C}-\mathrm{OH}$ & 289.4 & 1.35 & 6.4 & 289.5 & 1.3 & 0.85 & 289.1 & 1 & 0.96 & 289.4 & 1.35 & 1.1 \\
\hline
\end{tabular}

\begin{tabular}{|c|c|c|c|c|c|c|c|c|c|c|c|c|}
\hline \multicolumn{2}{|l|}{$01 \mathrm{~s}$} & \multicolumn{2}{|l|}{$\mathrm{OCC}$} & \multicolumn{3}{|c|}{$\mathrm{C}$} & \multicolumn{3}{|c|}{ C150 } & \multicolumn{3}{|c|}{ C1500CC } \\
\hline Sample & $\begin{array}{l}\mathrm{BE}, \\
\mathrm{eV}\end{array}$ & $\begin{array}{c}\text { FWHM, } \\
\text { eV }\end{array}$ & $\begin{array}{l}\mathrm{AC}, \\
\text { at.\% }\end{array}$ & $\begin{array}{l}\text { BE, } \\
\text { eV }\end{array}$ & $\begin{array}{c}\text { FWHM, } \\
\text { eV }\end{array}$ & $\begin{array}{l}\mathrm{AC}, \\
\text { at.\% }\end{array}$ & $\begin{array}{l}\text { BE, } \\
\text { eV }\end{array}$ & $\begin{array}{c}\text { FWHM, } \\
\text { eV }\end{array}$ & $\begin{array}{l}\text { AC, } \\
\text { at.\% }\end{array}$ & $\begin{array}{l}\text { BE, } \\
\text { eV }\end{array}$ & $\begin{array}{c}\text { FWHM, } \\
\text { eV }\end{array}$ & $\begin{array}{l}\mathrm{AC}, \\
\text { at.\% }\end{array}$ \\
\hline $\begin{array}{l}\mathrm{Si}-\mathrm{O} / \\
\mathrm{O}=\mathrm{C}-\mathrm{OH}\end{array}$ & 532.2 & 1.35 & 4.72 & 532.1 & 1.3 & 2.04 & 531.4 & 1.4 & 4.19 & 531.7 & 1.7 & 2.47 \\
\hline $\mathrm{C}-\mathrm{OH}$ & 533.6 & 1.95 & 15.48 & 533.2 & 1.5 & 30.25 & 533.0 & 1.5 & 18.84 & 533.2 & 1.5 & 28.94 \\
\hline Si $2 p$ & & OCC & & & C & & & C150 & & & C1500C & \\
\hline Sample & $\begin{array}{l}\mathrm{BE}, \\
\mathrm{eV}\end{array}$ & $\begin{array}{c}\text { FWHM, } \\
\text { eV }\end{array}$ & $\begin{array}{l}\mathrm{AC}, \\
\text { at.\% }\end{array}$ & $\begin{array}{l}\mathrm{BE}, \\
\mathrm{eV}\end{array}$ & $\begin{array}{c}\text { FWHM, } \\
\mathrm{eV}\end{array}$ & $\begin{array}{c}\mathrm{AC}, \\
\text { at.\% }\end{array}$ & $\begin{array}{l}\text { BE, } \\
\text { eV }\end{array}$ & $\begin{array}{c}\text { FWHM, } \\
\text { eV }\end{array}$ & $\begin{array}{l}\mathrm{AC}, \\
\text { at.\% }\end{array}$ & $\begin{array}{l}\mathrm{BE}, \\
\mathrm{eV}\end{array}$ & $\begin{array}{c}\text { FWHM, } \\
\text { eV }\end{array}$ & $\begin{array}{l}\mathrm{AC}, \\
\text { at.\% }\end{array}$ \\
\hline $\mathrm{Si}-\mathrm{O}$ & 102.3 & 1.25 & 0.41 & 102.3 & 1.25 & 2.94 & 102.3 & 1.3 & 2.12 & 102.2 & 1.35 & 1.37 \\
\hline
\end{tabular}




\begin{tabular}{|c|c|c|c|c|c|c|c|c|c|c|c|c|}
\hline N 1s & & OCC & & & C & & & C150 & & & C1500C & \\
\hline Sample & $\begin{array}{l}\text { BE, } \\
\text { eV }\end{array}$ & $\begin{array}{c}\text { FWHM, } \\
\text { eV }\end{array}$ & $\begin{array}{l}\mathrm{AC}, \\
\text { at.\% }\end{array}$ & $\begin{array}{l}\mathrm{BE}, \\
\mathrm{eV}\end{array}$ & $\begin{array}{c}\text { FWHM, } \\
\text { eV }\end{array}$ & $\begin{array}{l}\mathrm{AC}, \\
\text { at.\% }\end{array}$ & $\begin{array}{l}\text { BE, } \\
\text { eV }\end{array}$ & $\begin{array}{c}\text { FWHM, } \\
\text { eV }\end{array}$ & $\begin{array}{l}\mathrm{AC}, \\
\text { at.\% }\end{array}$ & $\begin{array}{l}\text { BE, } \\
\text { eV }\end{array}$ & $\begin{array}{c}\text { FWHM, } \\
\mathrm{eV}\end{array}$ & $\begin{array}{l}\mathrm{AC}, \\
\text { at.\% }\end{array}$ \\
\hline $\mathrm{N}\left(\mathrm{CH}_{3}\right)_{3}$ & & & & & & & 400.0 & 1.4 & 0.31 & 400.3 & 2.3 & 0.51 \\
\hline Org. N & 401.1 & 2.25 & 1.33 & & & & & & & & & \\
\hline $\mathrm{N}\left(\mathrm{CH}_{3}\right)_{3}{ }^{+}$ & & & & & & & 402.9 & 1.2 & 0.27 & 403.0 & 1.3 & 0.47 \\
\hline $\mathrm{NO}_{3}^{-}$ & 406.3 & 1.45 & 1.59 & & & & & & & 406.2 & 1.45 & 0.23 \\
\hline
\end{tabular}

Na 1s C150

\begin{tabular}{l|ccc}
\hline Sample & BE, eV & FWHM, eV & AC, at.\% \\
\hline $\mathrm{Na}^{+}$ & 1071.4 & 1.4 & 1.34 \\
\hline
\end{tabular}


3. Cationization reaction
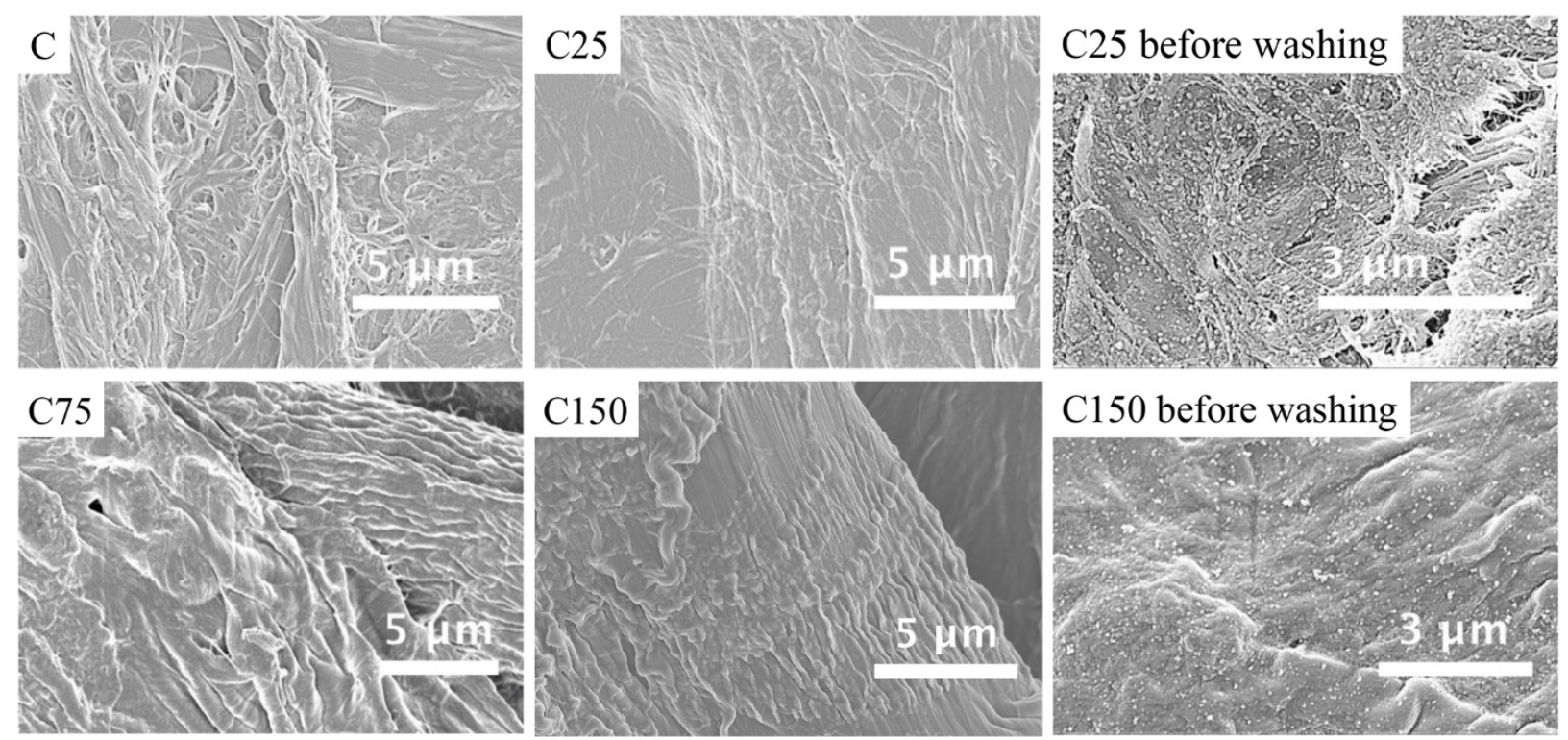

Figure S2. SEM images of C, C25, C75 and C150, and C25 and C150 before washing.

Table S2. Average nitrogen counts $\mathrm{mg}^{-1}$ of filters and experimental conditions applied for the cationization where $*=$ molar ratio.

\begin{tabular}{l|ccccc} 
Sample & $\begin{array}{c}\text { CHTAC } \\
\left(\mathrm{g} \mathrm{L}^{-1}\right)\end{array}$ & $\begin{array}{c}\mathrm{CHTAC} / \\
\mathrm{AGU} *\end{array}$ & $\begin{array}{c}\mathrm{NaOH} / \\
\mathrm{CHTAC} *\end{array}$ & $\begin{array}{c}\mathrm{NaOH} \text { excess } \\
\text { (M) }\end{array}$ & $\begin{array}{c}\text { Average nitrogen } \\
\text { counts } \mathrm{mg}^{-1}\end{array}$ \\
\hline C & n.a & n.a & n.a & n.a & $1.5 \times 10^{7}+/-2 \times 10^{6}$ \\
C25_low & 25 & 0.9 & 2.4 & 0.2 & $1.8 \times 10^{7}+/-1 \times 10^{7}$ \\
C25 & 25 & 0.9 & 4 & 0.4 & $2.5 \times 10^{7}+/-5 \times 10^{6}$ \\
C25_high & 25 & 0.9 & 10 & 1.2 & $2.0 \times 10^{7}+/-2 \times 10^{6}$ \\
C75 & 75 & 2.6 & 4 & 1.2 & $7.9 \times 10^{7}+/-2 \times 10^{7}$ \\
C150_low & 150 & 5.2 & 2.4 & 1.2 & $1.0 \times 10^{8}+/-3 \times 10^{7}$ \\
C150 & 150 & 5.2 & 4 & 2.4 & $1.1 \times 10^{8}+/-8 \times 10^{5}$ \\
C_NaOH & n.a & n.a & n.a & 3.2 & $8.3 \times 10^{6}+/-1 \times 10^{6}$ \\
\hline
\end{tabular}




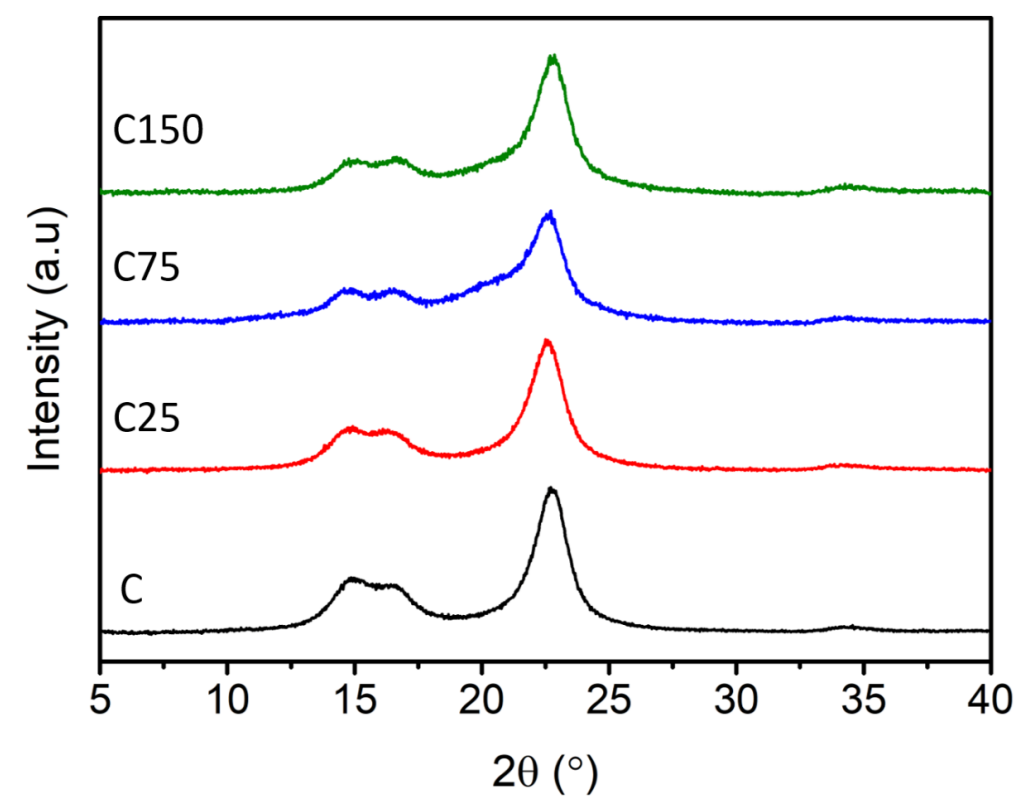

Figure S3. WAXD of C, C25, C75 and C150.

Table S3. Crystallinity index (CrI) of pristine cellulose C and cationized filters C25, C75 and C150.

\begin{tabular}{l|c} 
Sample & Crystallinity index (Crl) \\
\hline C & 0.90 \\
C25 & 0.87 \\
C75 & 0.83 \\
C150 & 0.86 \\
\hline
\end{tabular}

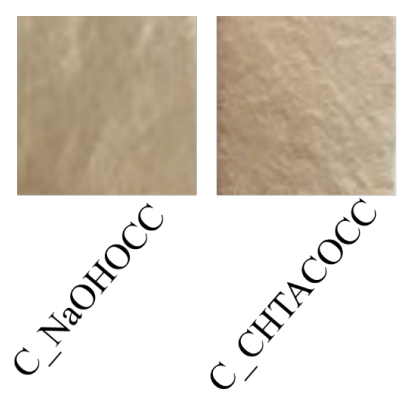

Figure S4. Images of C_NaOH and C_CHTAC after adsorption of OCC. 


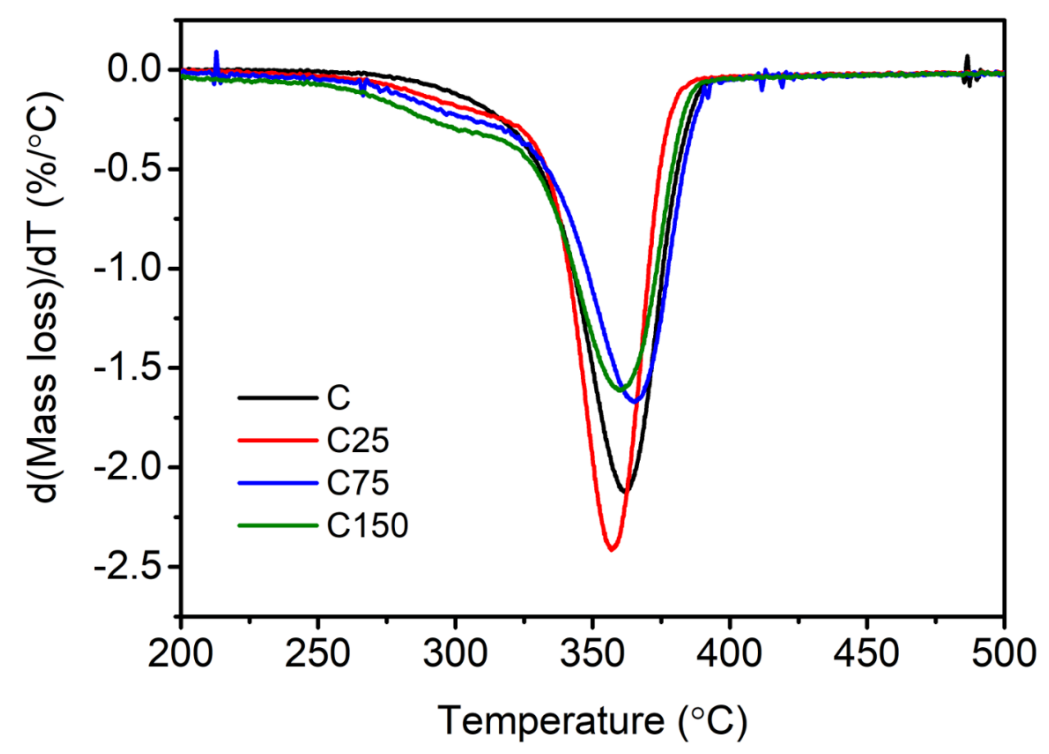

Figure S5. DTGA curve of filters before and after cationization.

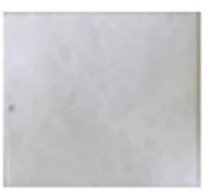

C

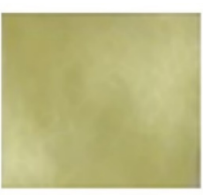

C150

Figure S6. Images of C and C150 filters after adsorption of potassium ferricyanide anion. 
4. OCC coated filters

Table S4. Average nitrogen counts $\mathrm{mg}^{-1}$ of OCC and OCC coated filters.

\begin{tabular}{l|c} 
Sample & Average nitrogen counts $\mathrm{mg}^{-1}$ \\
\hline OCC & $5.3 \times 10^{8}+/-4 \times 10^{7}$ \\
COCC & $2.5 \times 10^{7}+/-4 \times 10^{6}$ \\
C250CC & $7.0 \times 10^{7}+/-2 \times 10^{7}$ \\
C750CC & $1.0 \times 10^{8}+/+3 \times 10^{6}$ \\
C1500CC & $1.6 \times 10^{8}+/+1 \times 10^{7}$ \\
\hline
\end{tabular}

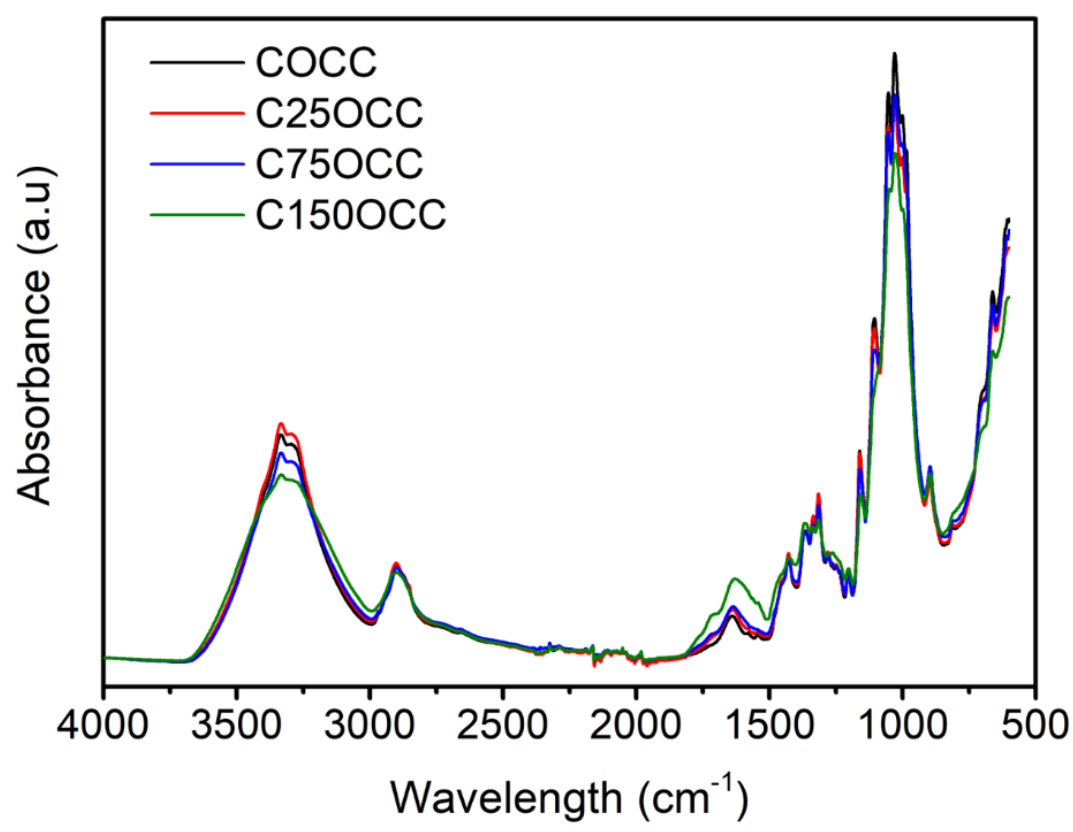

Figure S7. FTIR spectra of OCC coated filters. 
Table S5. Kinetic model parameters for filters with a MB feed concentration of $5 \mu \mathrm{g} \mathrm{mL} \mathrm{L}^{-1}$.

\begin{tabular}{l|c|c|c|c|c|c} 
Kinetic model & \multicolumn{3}{|c|}{ Pseduo-first order } & \multicolumn{3}{c}{ Pseudo-second order } \\
\hline Parameters & $k_{1}\left(\mathrm{~h}^{-1}\right)$ & $q_{e}\left(\mathrm{mg} \mathrm{g}^{-1}\right)$ & $R^{2}$ & $k_{2}\left(\mathrm{~g} \mathrm{mg}^{-1} \mathrm{~h}^{-1}\right)$ & $q_{e}\left(\mathrm{mg} \mathrm{g}^{-1}\right)$ & $R^{2}$ \\
\hline C-MB & 0.136 & 0.955 & 0.600 & 2.22 & 2.57 & 0.999 \\
C150-MB & 0.135 & 0.260 & 0.429 & 3.43 & 0.322 & 0.941 \\
C1500CC-MB & 0.249 & 1.90 & 0.843 & 0.464 & 3.71 & 0.998 \\
\hline
\end{tabular}

5. Additional SALDI-MS measurements

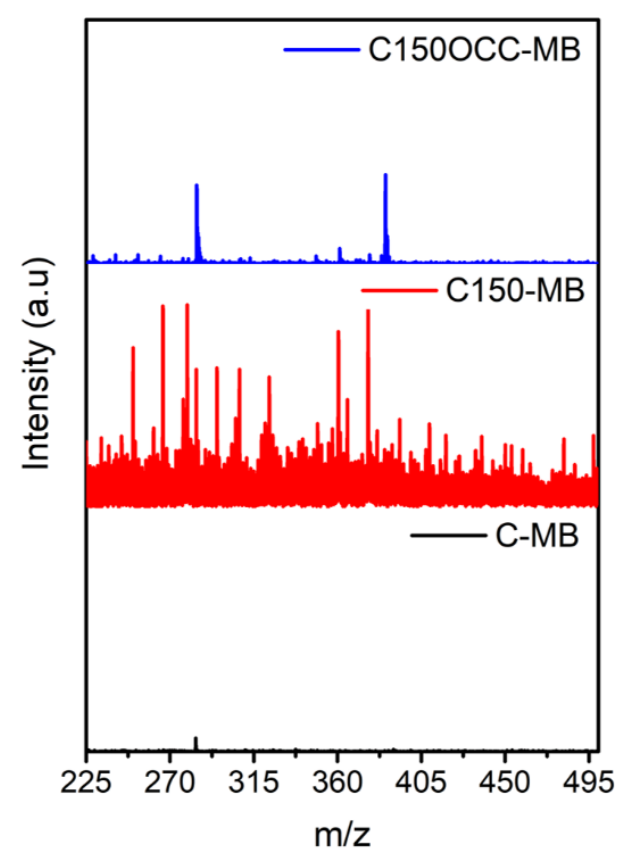

Figure S8. SALDI-MS of MB on the filters C, $\mathrm{C} 150$ and $\mathrm{C} 150 \mathrm{OCC}$ without $\mathrm{NaH}_{2} \mathrm{PO}_{4}$. 